\title{
Identification of potential biomarkers for pathogenesis of Alzheimer's disease
}

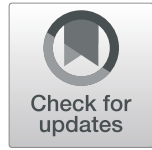

Huimin Wang ${ }^{1+}$, Xiujiang $\mathrm{Han}^{2+}$ and Sheng Gao ${ }^{2^{*}}$

\begin{abstract}
Background: Alzheimer's disease (AD) is an extremely complicated neurodegenerative disorder, which accounts for almost $80 \%$ of all dementia diagnoses. Due to the limited treatment efficacy, it is imperative for AD patients to take reliable prevention and diagnosis measures. This study aimed to explore potential biomarkers for AD.

Methods: GSE63060 and GSE140829 datasets were downloaded from the Gene Expression Omnibus (GEO) database. The differentially expressed genes (DEG) between AD and control groups in GSE63060 were analyzed using the limma software package. The mRNA expression data in GSE140829 was analyzed using weighted gene co-expression network analysis (WGCNA) function package. Protein functional connections and interactions were analyzed using STRING and key genes were screened based on the degree and Maximal Clique Centrality (MCC) algorithm. Gene Ontology (GO) and Kyoto Encyclopedia of Genes and Genomes (KEGG) enrichment analyses were performed on the key genes.

Results: There were 65 DEGs in GSE63060 dataset between AD patients and healthy controls. In GSE140829 dataset, the turquoise module was related to the pathogenesis of $A D$, among which, 42 genes were also differentially expressed in GSE63060 dataset. Then 8 genes, RPS17, RPL26, RPS3A, RPS25, EEF1B2, COX7C, HINT1 and SNRPG, were finally screened. Additionally, these 42 genes were significantly enriched in 12 KEGG pathways and $119 \mathrm{GO}$ terms.
\end{abstract}

Conclusions: In conclusion, RPS17, RPL26, RPS3A, RPS25, EEF1B2, COX7C, HINT1 and SNRPG, were potential biomarkers for pathogenesis of $A D$, which should be further explored in $A D$ in the future.

Keywords: Alzheimer's disease, Differentially expressed genes, Weighted gene co-expression network analysis, Biomarker

\section{Introduction}

Alzheimer's disease (AD) is an extremely complicated neurodegenerative disorder [1], which is usually characterized by progressive decline of abilities to varying degrees, such as memory, language, behavior and so on [2]. Additionally, AD accounts for almost $80 \%$ of all dementia diagnoses and has been the domain cause of dementia [3]. There are more than 45 million people

\footnotetext{
* Correspondence: tjgaosheng@outlook.com

${ }^{\dagger}$ Huimin Wang and Xiujiang Han equal contribution.

${ }^{2}$ Department of Geriatrics, Tianjin Hospital of ITCWM Nankai Hospital, No.6 Changjiang Road, Nankai, 300100 Tianjin, China

Full list of author information is available at the end of the article
}

worldwide suffering from $\mathrm{AD}$ and the number is estimated to approximate 131 million in next few decades according to a recent report [4]. On the one hand, the increasing number of AD patients is probably caused by the pathological heterogeneity characteristic of $\mathrm{AD}$ patients [5]. On the other hand, it has been widely reported that there are two pathologies in AD: $\beta$-amyloid plaque deposition and neurofibrillary tangles of hyperphosphorylated tau [6-8], however, no universally acceptable hypothesis could explain the pathogenesis of $\mathrm{AD}[1,9]$. Accordingly, despite the significant advancements in molecular medicine, the current $\mathrm{AD}$ treatments are still not enough to prevent the patients from the

\section{BMC}

(C) The Author(s). 2021 Open Access This article is licensed under a Creative Commons Attribution 4.0 International License, which permits use, sharing, adaptation, distribution and reproduction in any medium or format, as long as you give appropriate credit to the original author(s) and the source, provide a link to the Creative Commons licence, and indicate if changes were made. The images or other third party material in this article are included in the article's Creative Commons licence, unless indicated otherwise in a credit line to the material. If material is not included in the article's Creative Commons licence and your intended use is not permitted by statutory regulation or exceeds the permitted use, you will need to obtain permission directly from the copyright holder. To view a copy of this licence, visit http://creativecommons.org/licenses/by/4.0/ The Creative Commons Public Domain Dedication waiver (http://creativecommons.org/publicdomain/zero/1.0/) applies to the data made available in this article, unless otherwise stated in a credit line to the data. 
irreversible and progressive cognitive decline $[1,10]$, the negative influence of which is increasing as the aging population in many countries [11]. Collectively, it will be of great significance for all $\mathrm{AD}$ patients to take reliable prevention and diagnosis measures in earlier stage. Obviously, potential molecular biomarkers are helpful tools for AD prevention and diagnosis.

At present, it is imperative for those mild cognitive impairment (MCI) and AD patients to receive timely detection, early diagnosis and appropriate management [12]. Lots of researchers devoted to find reliable evidences for the understanding of the molecular pathogenesis of $\mathrm{AD}$, some of which have been transformed into promising treatment approaches [13, 14]. For instance, many biomarkers in cerebrospinal fluid (CSF) has been widely investigated, including amyloid- $\beta$ (A $\beta)[15,16]$, total tau levels $[17,18]$, phosphorylated tau levels [18, 19] and other novel candidate biomarkers. Moreover, it has been demonstrated that more than one biomarker would be more accurate to reveal the probability of $\mathrm{AD}$ due to mild cognitive impairment (MCI) [20]. Mean while, some reports revealed that CSF and PET biomarkers were reserved for some certain type of $\mathrm{AD}$ in clinical cases, like atypical, rapidly progressive $\mathrm{AD}$ and so on $[21,22]$. However, due to the invasiveness of CSF collection, this approach is greatly limited in the clinical application [14]. More researches began to explore other biomarkers from easily accessible fluid like plasma and urine. It has been reported that MT1 and several other genes were potential targets for $\mathrm{AD}$ therapy, but only weighted gene co-expression network analysis (WGCN A) was included in the study [6]. Another research has suggested that GRIK1 was related to AD stages, via WGCNA analysis [23]. Many previous studies usually focused on one certain gene or one analysis method. Therefore, except for WGCNA, other methods were also used in this research in order to find multiple potential biomarkers associated with the onset of $\mathrm{AD}$, so as to provide more reference information for $A D$ research in the future.

In the present study, two datasets GSE63060 and GSE140829 were downloaded from Gene Expression Omnibus (GEO, https://www.ncbi.nlm.nih.gov/geo/) database, and WGCNA, differential expression analysis and further analysis were integrated in our research. We herein aimed to screen novel potential biomarkers for $\mathrm{AD}$ patients through a comprehensive analysis.

\section{Materials and methods Data sources}

All mRNA expression data of Alzheimer's Disease (AD) was downloaded from Gene Expression Omnibus (GEO, https://www.ncbi.nlm.nih.gov/geo/) database. The GSE63060 dataset [24] included 145 blood samples from
$\mathrm{AD}$ patients and 104 blood samples from healthy controls. The mRNA expression data of this dataset was detected using the Illumina HumanHT-12 V3.0 expression beadchip platform. Another dataset GSE140829 included 204 blood samples from AD patients and 249 blood samples from healthy controls. The mRNA expression data of GSE140829 dataset was detected using HumanHT-12 v4 Expression BeadChip platform. The clinical characteristics of the samples in these two datasets were shown in Table S1.

\section{Differential expression analysis}

The differentially expressed genes (DEG) between $A D$ and control groups were analyzed using the limma software package [25] in $\mathrm{R}$ language. The $\left|\log _{2} \mathrm{FC}\right|>0.5$ and adjusted $P$ value $<0.05\left(\mathrm{P}_{\mathrm{FDR}}<0.05\right)$ after multiple testing by Benjamini and Hochberg $(\mathrm{BH})$ method were used as the standards to screen the DEGs.

\section{Weighted gene co-expression network analysis}

The mRNA expression data in GSE140829 was analyzed using WGCNA function package [26] in the R language. Via this method, all genes would be hierarchically clustered according to the gene expression value, then modules were identified using dynamic tree cutting method and those genes with higher similarity would be classified into the same module. Subsequently, the Module Eigengene (ME) value of each module was calculated. Additionally, the correlation coefficient between the ME value and certain phenotypes (such as type of disease, gender, age and so on) was calculated. When $p$ value was less than 0.05 , the larger the correlation coefficient, the closer relationship between this module and the phenotype.

\section{Functional enrichment analysis}

Gene ontology (GO) and Kyoto Encyclopedia of Genes and Genomes (KEGG) pathway enrichment analyses were performed using clusterProfiler [27] package in $R$ language. GO enrichment analysis included Biological Process (BP), Cellular Component (CC) and Molecular Function (MF) terms. The GO terms and KEGG pathways with $P$. adjust $<0.05$ were considered to be significantly enriched.

\section{Protein-protein interaction (PPI) network analysis}

STRING is a database used for protein functional connection and protein-protein interaction analyses. Here, protein functional connections and interactions were analyzed using STRING (https://string-db.org/,version 11.0) [28]. The interaction pairs with confidence score $\geq$ 0.4 were retained. The PPI network was then visualized using Cytoscape (version 3.7.2) (https://cytoscape.org/) [29]. The key genes in PPI network were screened based 
on the degree of nodes and Maximal Clique Centrality (MCC) algorithm, using cytoHubba plug-in of Cytoscape software.

\section{Results}

\section{Differentially expressed genes}

Based on all mRNA expression data in GSE63060 dataset, the DEGs between $\mathrm{AD}$ and healthy control groups were identified. Between AD patients and healthy controls, there were 65 DEGs, including 1 up-regulated gene and 64 down-regulated genes (Fig. 1A). The expression levels of these DEGs were significantly different between the two groups (Fig. 1B).

\section{Potential genes related to $A D$ occurrence identified via WGCNA analysis}

WGCNA analysis was performed on the samples in GSE140829 dataset. The results showed that the gene co-expression network conformed to unsigned network. The higher the square of the correlation coefficient, the closer the network was to the distribution of unsigned network. The square of the correlation coefficient, 0.85 , was taken as the standard to select the soft threshold $(\beta=10$, Fig. 2A).

Then the genes were clustered based on averagelinkage hierarchical clustering method. According to the standard of dynamic tree cutting, 100 was set as the minimum number of genes in each gene network module. Subsequently, module eigengene (ME) value of each module was calculated in turn and cluster analysis was performed on the modules. The close modules were merged into a new one. With the height of $0.25,6$ modules were finally obtained (Fig. 2B). The gray module contained all genes that could not be clustered in other modules.
According to the ME values of the obtained modules, the correlations between these modules and the phenotypes were calculated. The results suggested that the correlation between the turquoise module and sample type was the largest (correlation coefficient was -0.16 , $P=8 \mathrm{e}-4$ ) (Fig. 2C), which indicated that the genes in turquoise module $(n=2215)$ obtained via WGCNA were potentially related to the pathogenesis of AD. Moreover, 42 of the 2215 genes in turquoise module were also differentially expressed in GSE63060 dataset (both were up-regulated in AD), which implied that these 42 genes were potentially more associated with the pathogenesis of $\mathrm{AD}$ (Fig. 2D).

\section{Significantly enriched GO terms and KEGG pathways}

In order to obtain more function information of the 42 shared genes, GO and KEGG pathway enrichment analyses were performed. GO terms were significantly enriched, including 51 Biological Process terms, 52 Cellular Component terms and 16 Molecular Function terms. The top 10 significantly enriched Biological Process terms (Fig. 3A), Cellular Component terms (Fig. 3B) and Molecular Function terms (Fig. 3C) were displayed in Fig. 3A-C. There were 12 KEGG pathways that were significantly enriched (Fig. 3D). The detailed results of GO and KEGG pathway enrichment analyses were shown in Table $\mathrm{S} 2$.

\section{PPI network construction and key genes screening}

The 42 genes were chosen to construct the PPI network. The interaction pairs with confidence score $\geq 0.4$ were visualized using Cytoscape software (Fig. 4A). Among the 42 genes, there were 39 interacting genes. Among them, COX7C had the highest node degree of 26 , and the lowest node degree was 13 . The top 10 genes screened according to the degree of the node were
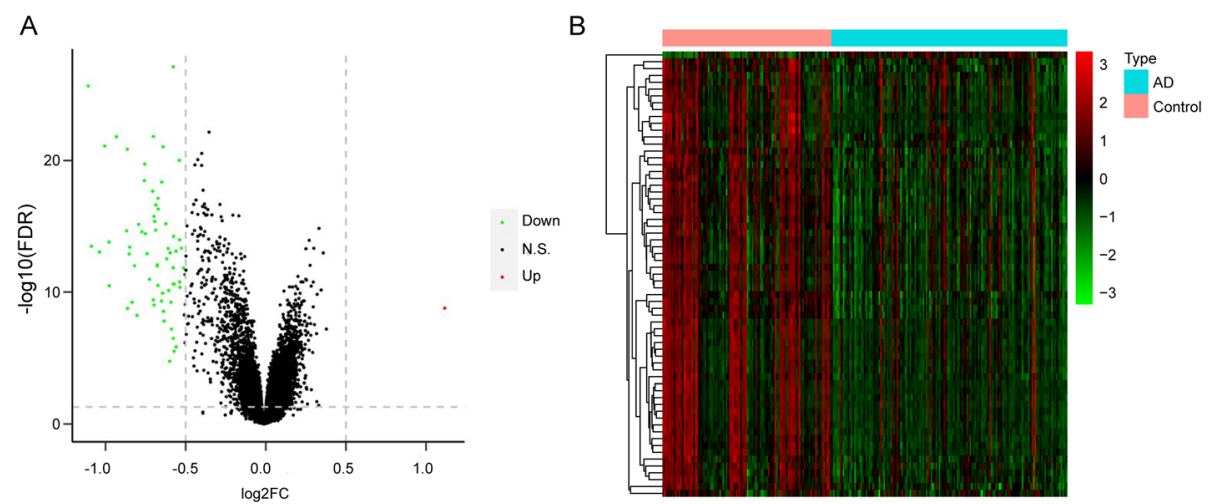

Fig. 1 Differentially expressed genes. A Volcano map of differentially expressed genes in GSE63060 dataset. Horizontal axis: the log 2 FC value; vertical axis: - $\log 10$ (FDR). Red: up-regulation; green: down-regulation; black: non-significant difference. B Expression level heat map of differentially expressed genes in GSE63060. Horizontal axis: genes; vertical axis: samples. Red: high expression; green: low expression 

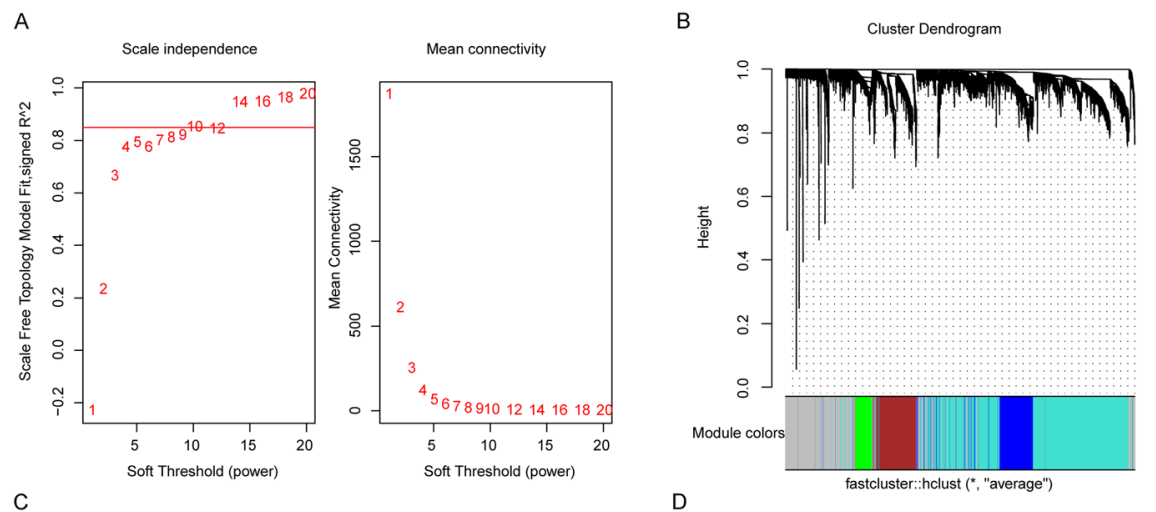

$\mathrm{C}$
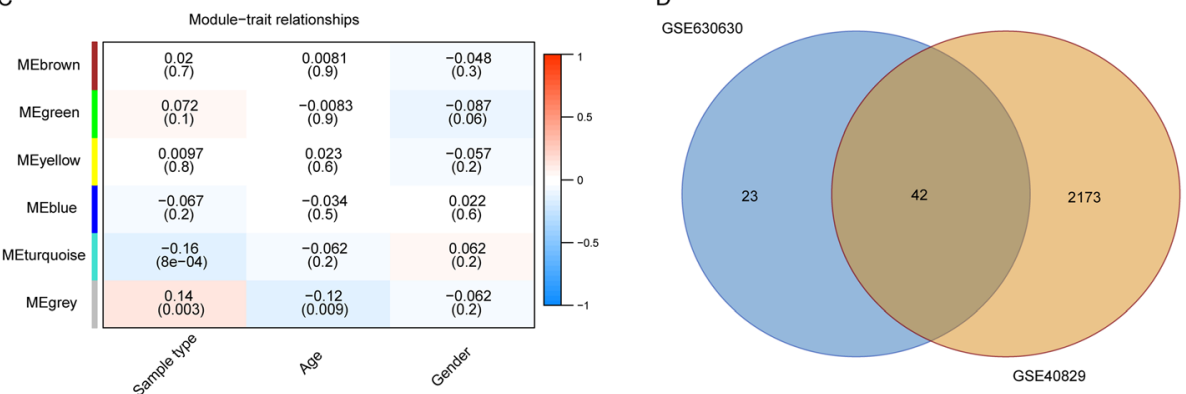

Fig. 2 Results of WGCNA. A Schematic diagram of soft threshold screening. Red line represented the square of the correlation coefficient, 0.85 , and the first point above red line was the soft threshold $\beta=10$. B Schematic diagram of gene module clustering. Each color represented a module, and the gray module included the genes that could not be clustered into any module. $\mathbf{C}$ Heat map of the correlation between gene module and phenotype. Red: positive correlation; blue: negative correlation. The darker the color, the greater the correlation. D Venn diagram of differentially expressed genes in GSE63060 dataset and genes screened via WGCNA in GSE140829 dataset
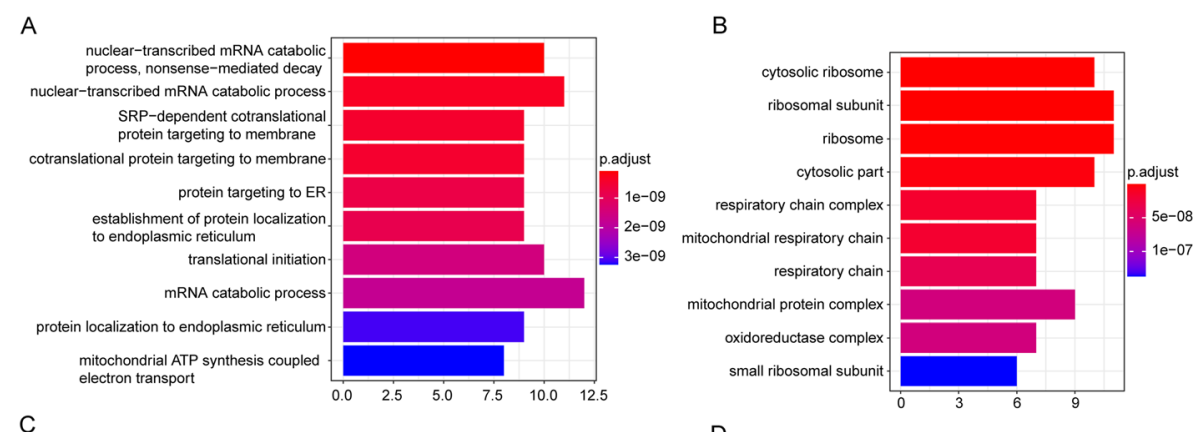

C

D

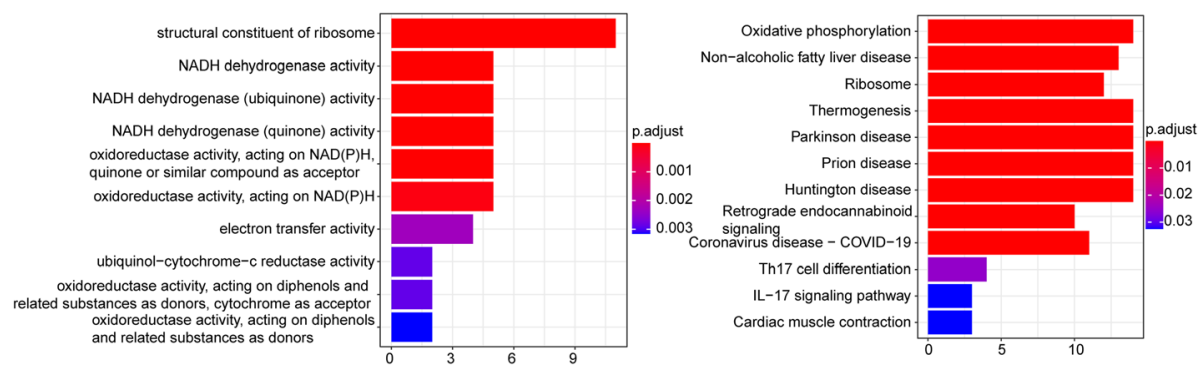

Fig. 3 Significantly enriched GO terms and KEGG pathways.A The top 10 significantly enriched Biological Process terms. B The top 10 significantly enriched Cellular Component terms. C The top 10 significantly enriched Molecular Function terms. D The 12 significantly enriched KEGG pathways. Horizontal axis: the number of enriched genes; vertical axis: the corresponding biological process; different p.adjust values were represented by altered colors 


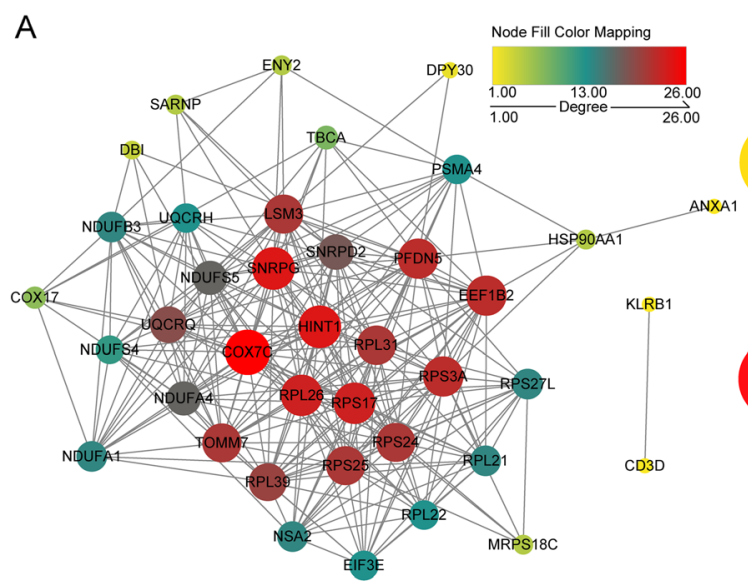

B

C

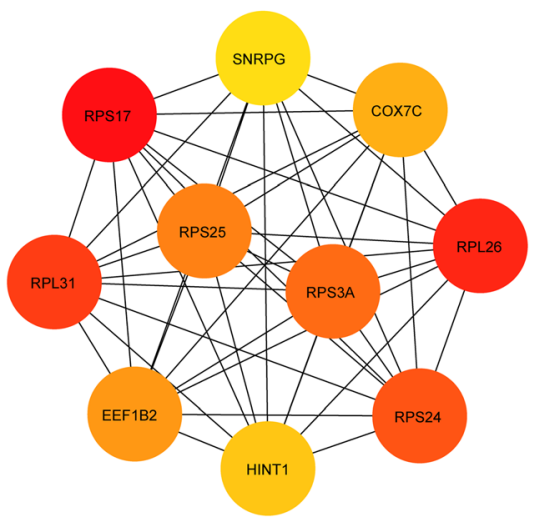

Fig. 4 PPI network construction and key genes screening. A Diagram of protein-protein interaction network. Each dot represented a node. The more line segments connected to the node, the greater the degree of this node. The degree of node was reflected by its size and color. The larger the node, the deeper color from yellow to red, the greater the degree. B Network diagram of the top 10 genes screened according to degree. The darker color from yellow to red represented greater degree. $\mathbf{C}$ The network diagram of the top 10 genes screened via MCC algorithm. The darker color from yellow to red represented greater score

displayed in Fig. 4B, using cytoHubba plug-in of Cytoscape software. The top 10 genes screened according to the MCC algorithm were shown in Fig. 4C, among which, 8 genes were overlapped with the top 10 genes screened by the degree. The results suggested that these 8 genes, including RPS17, RPL26, RPS3A, RPS25, EEF1B2, COX7C, HINT1 and SNRPG, were more crucial to the pathogenesis of AD. Detailed degree and MCC algorithm score of these 8 genes were displayed in Table 1.

Additionally, as differentiating AD from other diseases with similar symptoms is of great clinical significance, we also established a logistic regression model based on the selected 8 genes to further evaluate our biomarkers in distinguishing $\mathrm{AD}$ from mild cognitive impairment (MCI), another type of dementia disorder. As shown in Figure S1, the area under curve (AUC) value of the established model was 0.6021 , indicating that the model based on the selected genes had potential value in differentiating AD from MCI. However, this is only a preliminary research on the utility of the selected genes, and further optimization is needed for their pervasive application in this regard.

Table 1 The degree and MCC score of the selected 8 genes

\begin{tabular}{lcc}
\hline Gene & Degree & MCC score \\
\hline RPS17 & 23 & $1.05 \mathrm{E}+09$ \\
RPL26 & 23 & $1.05 \mathrm{E}+09$ \\
RPS3A & 22 & $1.04 \mathrm{E}+09$ \\
RPS25 & 21 & $1.04 \mathrm{E}+09$ \\
EEF1B2 & 22 & $1.01 \mathrm{E}+09$ \\
COX7C & 26 & $9.68 \mathrm{E}+08$ \\
HINT1 & 24 & $9.64 \mathrm{E}+08$ \\
SNRPG & 24 & $9.63 \mathrm{E}+08$ \\
\hline
\end{tabular}




\section{Discussion}

In this research, via an integrated analysis including WGCNA, differential expression analysis and other further analyses of the data downloaded from GEO database, we have explored the possible biomarkers for AD patients. There were 65 DEGs between AD patients and healthy controls in GSE63060 dataset. Subsequently, via WGCNA, the turquoise module was found to be related to the pathogenesis of AD. Finally, 8 genes, including RPS17, RPL26, RPS3A, RPS25, EEF1B2, COX7C, HINT1 and SNRPG, were evidenced to be crucial to the pathogenesis of $\mathrm{AD}$.

Two datasets, GSE63060 and GSE140829, were included in our research. Firstly, in GSE63060 dataset, 65 genes were found to be differentially expressed between AD patients and healthy controls, including 1 upregulated gene and 64 down-regulated genes. Then, WGCNA analysis was performed on the samples in GSE140829 dataset, through which, 6 co-expression modules were obtained and the turquoise module was related to the pathogenesis of AD. Among all 2215 genes in turquoise module, 42 genes were also differentially expressed in GSE63060 dataset. As far as we know, most of the previous studies only used WGCNA to find $\mathrm{AD}$ related genes $[6,30]$, but we have integrated the results of DEGs and WGCNA in order to obtain a more reliable basis before further analysis. Based on the PPI network, 8 genes among all 42 genes were finally screened according to the degree and MCC algorithm, which included RPS17, RPL26, RPS3A, RPS25, EEF1B2, COX7C, HINT1 and SNRPG. Some direct or indirect evidence was found to support most of the 8 genes. Regarding RPL26, Mastroeni at al. suggested that it was a marker of ribosome in neurons and could be used in methylation related studies in AD neurons in the future [31]. And a recent research demonstrated that RPS3A was not only major pathogenic gene of $\mathrm{MCI}$, but bridge gene; SNRPG was not only major pathogenic gene of $\mathrm{MCI}$ and $\mathrm{AD}$, but also bridge gene [32]. Our results were consistent with the previous researches to some extent. It has been suggested that RPS25 might be a potential therapeutic target for C9orf72-related neurodegenerative diseases caused by nucleotide repeat expansions [33], which could support our findings indirectly. A study documented that AEEF1B2 belonged to the eukaryotic elongation translational machinery and AEEF1B2 variants in the translational machinery were associated with several neurodevelopmental disorders [34]. It has been reported that the expression of COX7C was decreased in total homogenates of the entorhinal cortex in AD stages V-VI [35]. However, RPS17 and HINT1 have not been studied in AD, which should be investigated in the future. Collectively, these 8 genes were potential biomarkers for pathogenesis of
$\mathrm{AD}$ and they could be further explored in $\mathrm{AD}$ researches.

In addition, GO and KEGG pathway enrichment analyses were performed on the 42 shared genes in order to obtain more function information of them. GO terms were significantly enriched, including 51 Biological Process terms, 52 Cellular Component terms and 16 Molecular Function terms. The 42 genes were significantly enriched in 12 KEGG pathways, including Oxidative phosphorylation, Nonalcoholic fatty liver disease (NAFLD), Parkinson disease and several other pathways. Some of the KEGG pathways were related to the previous studies to some extent and they partially provided new ideas for future researches. For example, several studies have demonstrated the complicated roles of oxidative stress in the pathogenesis and progression of AD [36-38], which could be linked to the oxidative phosphorylation pathway in further studies. Regarding Non-alcoholic fatty liver disease (NAFLD) pathway, it has been reported that NAFLD-induced chronic inflammation induced neurodegeneration diseases in wild-type mice [39], which could also be correlated with inflammation in $\mathrm{AD}$ as inflammation had been evidenced to be associated with the pathogenesis of $\mathrm{AD}[40,41]$. In addition, some genes were significantly enriched in Parkinson disease pathway, which indicated that Parkinson disease and AD might be explored together, as age-related neurodegeneration diseases [42, 43]. Collectively, these pathways have provided more information for the further exploration of AD in the near future and we would also spare no effort to continue the researches on AD.

\section{Conclusions}

In conclusion, via integrating the results of WGCNA and DEGs, 8 genes, including RPS17, RPL26, RPS3A, RPS25, EEF1B2, COX7C, HINT1 and SNRPG, were further screened and evidenced to be associated with the occurrence of AD. These genes were significantly enriched in Oxidative phosphorylation, NAFLD and some other pathways. Our findings will provide more information for this complicated neurodegenerative disorder.

\footnotetext{
Abbreviations

AD: Alzheimer's disease; GEO: Gene Expression Omnibus ; DEG: Differentially expressed genes; WGCNA: Weighted gene co-expression network analysis ; MCC: Maximal Clique Centrality ; GO: Gene Ontology ; KEGG: Kyoto Encyclopedia of Genes and Genomes ; MCl: Mild cognitive impairment ; CSF: Cerebrospinal fluid ; ME : Module Eigengene ; BP: Biological Process CC: Cellular Component ; MF: Molecular Function ; PPI: Protein-protein interaction ; AUC: Area under curve ; NAFLD: Non-alcoholic fatty liver disease
} 


\section{Supplementary Information}

The online version contains supplementary material available at https:/doi. org/10.1186/s41065-021-00187-9.

Additional file 1: Figure S1. Establishment of the logistic regression model based on the selected 8 genes. (A) Residuals vs leverage plot to detect the influential point. The red dashed line indicated the COOK distance. The point with a COOK distance greater than 0.05 was considered as an influential point, which could affect the reliability of the model. It was shown that there were no influential points for our model. (B) Component plus residual plots of the selected 8 genes. The obvious linear relationship between the horizontal axis and vertical axis indicated that the independent variable could be included in the model. (C) The ROC curve. The horizontal axis denoted the false positive rate, and the vertical axis denoted the true positive rate.

Additional file 2. The clinical characteristics of samples in GSE63060 and GSE140829 datasets.

Additional file 3: Table S2. Significantly enriched GO entries and KEGG pathways.

\section{Acknowledgements}

Not applicable.

\section{Authors' contributions}

All authors contributed to the study conception and design. Data collection and analysis were performed by Xiujiang Han, Sheng Gao. The first draft of the manuscript was written by Huimin Wang. All authors read and approved the final manuscript.

\section{Funding}

Not applicable.

\section{Availability of data and materials}

The data that support the findings of this study are available in (GEO) at (https://www.ncbi.nlm.nih.gov/geo/).

\section{Declarations}

Ethics approval and consent to participate

Not applicable.

\section{Consent for publication}

Not applicable.

\section{Competing interests}

The authors declare that there are no conflicts of interest.

\section{Author details}

'Department of Neurology, Tianjin Hospital of ITCWM Nankai Hospital, 300100 Tianjin, China. ${ }^{2}$ Department of Geriatrics, Tianjin Hospital of ITCWM Nankai Hospital, No.6 Changjiang Road, Nankai, 300100 Tianjin, China.

Received: 31 January 2021 Accepted: 31 May 2021

Published online: 05 July 2021

\section{References}

1. Raikwar SP, Thangavel R, Dubova I, Ahmed ME, Selvakumar PG, Kempuraj D, Zaheer S, lyer S, Zaheer A. Neuro-Immuno-Gene- and Genome-EditingTherapy for Alzheimer's Disease: Are We There Yet? J Alzheimers Dis. 2018; 65(2):321-44.

2. Crous-Bou M, Minguillon C, Gramunt N, Molinuevo JL. Alzheimer's disease prevention: from risk factors to early intervention. Alzheimers Res Ther 2017;9(1):71.

3. Realdon O, Rossetto F, Nalin M, Baroni I, Cabinio M, Fioravanti R, Saibene FL, Alberoni M, Mantovani F, Romano M, et al. Technology-enhanced multidomain at home continuum of care program with respect to usual care for people with cognitive impairment: the Ability-TelerehABILITation study protocol for a randomized controlled trial. BMC Psychiatry. 2016;16(1):425.
4. Prince M, Ali GC, Guerchet M, Prina AM, Albanese E, Wu YT. Recent global trends in the prevalence and incidence of dementia, and survival with dementia. Alzheimers Res Ther. 2016;8(1):23.

5. Armstrong RA, Nochlin D, Bird TD. Neuropathological heterogeneity in Alzheimer's disease: a study of 80 cases using principal components analysis. Neuropathology. 2000;20(1):31-7.

6. L Liang JW, Fang ZY, Huang Y, Liuyang ZY, Zhang XL, Wang JL, Wei H, Wang JZ, Wang XC, Zeng J, et al. Application of Weighted Gene Co-Expression Network Analysis to Explore the Key Genes in Alzheimer's Disease. J Alzheimers Dis. 2018;65(4):1353-64.

7. Livingston G, Sommerlad A, Orgeta V, Costafreda SG, Huntley J, Ames D, Ballard C, Banerjee S, Burns A, Cohen-Mansfield J, et al. Dementia prevention, intervention, and care. Lancet. 2017:390(10113):2673-734.

8. Koldamova R, Fitz NF, Lefterov I. The role of ATP-binding cassette transporter A1 in Alzheimer's disease and neurodegeneration. Biochim Biophys Acta. 2010;1801(8):824-30.

9. $\quad$ R AA. Risk factors for Alzheimer's disease. Folia Neuropathol. 2019;57(2):87-105.

10. Esquerda-Canals G, Montoliu-Gaya L, Guell-Bosch J, Villegas S. Mouse Models of Alzheimer's Disease. J Alzheimers Dis. 2017:57(4):1171-83.

11. Weller J, Budson A: Current understanding of Alzheimer's disease diagnosis and treatment. F1000Res. 2018;7. https:/pubmed.ncbi.nlm.nih.gov/30135715/.

12. 2020 Alzheimer's disease facts and figures. Alzheimers Dement. 2020. https://doi.org/10.1002/alz.12068. Epub ahead of print.

13. Wang YQ, Qu DH, Wang K. Therapeutic approaches to Alzheimer's disease through stimulating of non-amyloidogenic processing of amyloid precursor protein. Eur Rev Med Pharmacol Sci. 2016;20(11):2389-403.

14. Hane FT, Robinson M, Lee BY, Bai O, Leonenko Z, Albert MS. Recent Progress in Alzheimer's Disease Research, Part 3: Diagnosis and Treatment. J Alzheimers Dis. 2017;57(3):645-65.

15. Arbor SC, LaFontaine M, Cumbay M. Amyloid-beta Alzheimer targets protein processing, lipid rafts, and amyloid-beta pores. Yale J Biol Med. 2016;89(1):5-21.

16. Lei $Y$, Renyuan Z. Effects of Androgens on the Amyloid-beta Protein in Alzheimer's Disease. Endocrinology. 2018;159(12):3885-94.

17. Pase MP, Beiser AS, Himali JJ, Satizabal CL, Aparicio HJ, DeCarli C, Chene G, Dufouil C, Seshadri S. Assessment of Plasma Total Tau Level as a Predictive Biomarker for Dementia and Related Endophenotypes. JAMA Neurol. 2019; 76(5):598-606.

18. Rubenstein R, Chang B, Yue JK, Chiu A, Winkler EA, Puccio AM, Diaz-Arrastia R, Yuh EL, Mukherjee P, Valadka AB, et al. Comparing Plasma Phospho Tau, Total Tau, and Phospho Tau-Total Tau Ratio as Acute and Chronic Traumatic Brain Injury Biomarkers. JAMA Neurol. 2017;74(9):1063-72.

19. Reddy PH, Oliver DM. Amyloid Beta and Phosphorylated Tau-Induced Defective Autophagy and Mitophagy in Alzheimer's Disease. Cells. 2019;8(5):488.

20. Mantzavinos V, Alexiou A. Biomarkers for Alzheimer's Disease Diagnosis. Curr Alzheimer Res. 2017;14(11):1149-54.

21. Johnson KA, Minoshima S, Bohnen NI, Donohoe KJ, Foster NL, Herscovitch P, Karlawish JH, Rowe CC, Carrillo MC, Hartley DM, et al. Appropriate use criteria for amyloid PET: a report of the Amyloid Imaging Task Force, the Society of Nuclear Medicine and Molecular Imaging, and the Alzheimer's Association. J Nucl Med. 2013:54(3):476-90.

22. Simonsen AH, Herukka SK, Andreasen N, Baldeiras I, Bjerke M, Blennow K, Engelborghs S, Frisoni GB, Gabryelewicz T, Galluzzi S, et al. Recommendations for CSF AD biomarkers in the diagnostic evaluation of dementia. Alzheimers Dement. 2017:13(3):274-84.

23. Sun $Y$, Lin J, Zhang $L$. The application of weighted gene co-expression network analysis in identifying key modules and hub genes associated with disease status in Alzheimer's disease. Ann Transl Med. 2019;7(24):800.

24. Sood S, Gallagher IJ, Lunnon K, Rullman E, Keohane A, Crossland H, Phillips $B E_{1}$ Cederholm $T$, Jensen $T$, van Loon $L$, et al. A novel multi-tissue RNA diagnostic of healthy ageing relates to cognitive health status. Genome Biol. 2015:16:185

25. Ritchie ME, Phipson B, Wu D, Hu Y, Law CW, Shi W, Smyth GK. limma powers differential expression analyses for RNA-sequencing and microarray studies. Nucleic Acids Res. 2015;43(7):e47.

26. Zhang B, Horvath S. A general framework for weighted gene co-expression network analysis. Stat Appl Genet Mol Biol. 2005;4:Article17.

27. Yu G, Wang LG, Han Y, He QY. clusterProfiler: an R package for comparing biological themes among gene clusters. OMICS. 2012;16(5):284-7.

28. Szklarczyk D, Gable AL, Lyon D, Junge A, Wyder S, Huerta-Cepas J, Simonovic M, Doncheva NT, Morris JH, Bork P, et al. STRING v11: protein- 
protein association networks with increased coverage, supporting functional discovery in genome-wide experimental datasets. Nucleic Acids Res. 2019; 47(D1):D607-13.

29. Shannon P, Markiel A, Ozier O, Baliga NS, Wang JT, Ramage D, Amin N, Schwikowski B, Ideker T. Cytoscape: a software environment for integrated models of biomolecular interaction networks. Genome Res. 2003;13(11): 2498-504.

30. Soleimani Zakeri NS, Pashazadeh S, MotieGhader H. Gene biomarker discovery at different stages of Alzheimer using gene co-expression network approach. Sci Rep. 2020;10(1):12210.

31. Mastroeni D, Grover A, Delvaux E, Whiteside C, Coleman PD, Rogers J. Epigenetic changes in Alzheimer's disease: decrements in DNA methylation. Neurobiol Aging. 2010;31(12):2025-37.

32. Tao Y, Han Y, Yu L, Wang Q, Leng SX, Zhang H. The Predicted Key Molecules, Functions, and Pathways That Bridge Mild Cognitive Impairment (MCI) and Alzheimer's Disease (AD). Front Neurol. 2020;11:233.

33. Yamada SB, Gendron TF, Niccoli T, Genuth NR, Grosely R, Shi Y, Glaria I, Kramer NJ, Nakayama L, Fang S, et al. RPS25 is required for efficient RAN translation of C9orf72 and other neurodegenerative disease-associated nucleotide repeats. Nat Neurosci. 2019;22(9):1383-8.

34. Larcher L, Buratti J, Heron-Longe B, Benzacken B, Pipiras E, Keren B, Delahaye-Duriez A. New evidence that biallelic loss of function in EEF1B2 gene leads to intellectual disability. Clin Genet. 2020;97(4):639-43.

35. Armand-Ugon M, Ansoleaga B, Berjaoui S, Ferrer I. Reduced Mitochondrial Activity is Early and Steady in the Entorhinal Cortex but it is Mainly Unmodified in the Frontal Cortex in Alzheimer's Disease. Curr Alzheimer Res. 2017;14(12):1327-34.

36. Butterfield DA, Boyd-Kimball D. Oxidative Stress, Amyloid-beta Peptide, and Altered Key Molecular Pathways in the Pathogenesis and Progression of Alzheimer's Disease. J Alzheimers Dis. 2018;62(3):1345-67.

37. Vallee A, Lecarpentier $Y$, Guillevin R, Vallee JN. Effects of cannabidiol interactions with Wnt/beta-catenin pathway and PPARgamma on oxidative stress and neuroinflammation in Alzheimer's disease. Acta Biochim Biophys Sin (Shanghai). 2017;49(10):853-66.

38. Yan $\mathrm{MH}$, Wang $\mathrm{X}$, Zhu X. Mitochondrial defects and oxidative stress in Alzheimer disease and Parkinson disease. Free Radic Biol Med. 2013;62:90101.

39. Kim DG, Krenz A, Toussaint LE, Maurer K, Robinson SA, Yan A, Torres L, Bynoe MS. Non-alcoholic fatty liver disease induces signs of Alzheimer's disease (AD) in wild-type mice and accelerates pathological signs of AD in an AD model. J Neuroinflammation. 2016;13:1.

40. Sochocka M, Donskow-Lysoniewska K, Diniz BS, Kurpas D, Brzozowska E, Leszek J. The Gut Microbiome Alterations and Inflammation-Driven Pathogenesis of Alzheimer's Disease-a Critical Review. Mol Neurobiol. 2019; 56(3):1841-51

41. Holmes C. Review: systemic inflammation and Alzheimer's disease Neuropathol Appl Neurobiol. 2013;39(1):51-68

42. Wlodarek D. Role of Ketogenic Diets in Neurodegenerative Diseases (Alzheimer's Disease and Parkinson's Disease). Nutrients. 2019;11(1):169.

43. Belaidi AA, Bush Al. Iron neurochemistry in Alzheimer's disease and Parkinson's disease: targets for therapeutics. J Neurochem. 2016;139(Suppl 1):179-97.

\section{Publisher's Note}

Springer Nature remains neutral with regard to jurisdictional claims in published maps and institutional affiliations.

Ready to submit your research? Choose BMC and benefit from:

- fast, convenient online submission

- thorough peer review by experienced researchers in your field

- rapid publication on acceptance

- support for research data, including large and complex data types

- gold Open Access which fosters wider collaboration and increased citations

- maximum visibility for your research: over $100 \mathrm{M}$ website views per year

At $\mathrm{BMC}$, research is always in progress.

Learn more biomedcentral.com/submissions 